\title{
BMJ Open Current use of inotropes according to initial blood pressure and peripheral perfusion in the treatment of congestive heart failure: findings from a multicentre observational study
}

Kazuya Nagao (D) , , Takao Kato (D) , ${ }^{2}$ Hidenori Yaku, ${ }^{3}$ Takeshi Morimoto (D) ,4 Yasutaka Inuzuka, ${ }^{5}$ Yodo Tamaki, ${ }^{6}$ Erika Yamamoto, ${ }^{2}$ Yusuke Yoshikawa, ${ }^{2}$ Takeshi Kitai, ${ }^{7}$ Ryoji Taniguchi, ${ }^{8}$ Moritake Iguchi (D) , ${ }^{9}$ Masashi Kato, ${ }^{3}$ Mamoru Takahashi, ${ }^{10}$ Toshikazu Jinnai, ${ }^{11}$ Tomoyuki Ikeda, ${ }^{12}$ Takafumi Kawai, ${ }^{13}$ Akihiro Komasa, ${ }^{14}$ Ryusuke Nishikawa, ${ }^{15}$ Yuichi Kawase, ${ }^{16}$ Takashi Morinaga, ${ }^{17}$ Kanae Su, ${ }^{18}$ Mitsunori Kawato, ${ }^{19}$ Yuta Seko, ${ }^{2}$ Tsukasa Inada, ${ }^{1}$ Moriaki Inoko, ${ }^{20}$ Mamoru Toyofuku, ${ }^{18}$ Yutaka Furukawa, ${ }^{21}$ Yoshihisa Nakagawa, ${ }^{22}$ Kenji Ando, ${ }^{17}$ Kazushige Kadota, ${ }^{16}$ Satoshi Shizuta (D) , ${ }^{2}$ Koh Ono, ${ }^{2}$ Yukihito Sato, ${ }^{8}$ Koichiro Kuwahara, ${ }^{23}$ Neiko Ozasa, ${ }^{2}$ Takeshi Kimura (D) ${ }^{2}$

To cite: Nagao K, Kato T, Yaku $\mathrm{H}$, et al. Current use of inotropes according to initial blood pressure and peripheral perfusion in the treatment of congestive heart failure: findings from a multicentre observational study. BMJ Open 2022;12:e053254. doi:10.1136/ bmjopen-2021-053254

- Prepublication history and additional supplemental material for this paper are available online. To view these files, please visit the journal online (http://dx.doi.org/10.1136/ bmjopen-2021-053254)

Received 09 May 2021 Accepted 22 December 2021

Check for updates

(c) Author(s) (or their employer(s)) 2022. Re-use permitted under CC BY-NC. No commercial re-use. See rights and permissions. Published by BMJ.

For numbered affiliations see end of article.

Correspondence to

Dr Takao Kato;

tkato75@kuhp.kyoto-u.ac.jp

\section{ABSTRACT}

Objectives Current guidelines restrict the use of inotropes for the treatment for heart failure (HF) unless the patients are hypotensive or hypoperfused because of safety concerns. This study sought to characterise the contemporary real-world use of inotropes and associated long-term outcomes according to systolic blood pressure (sBP) and perfusion status.

Design A multicentre prospective cohort study.

Setting This study was nested from the Kyoto Congestive Heart Failure registry, which included consecutive Japanese patients admitted for HF.

Participants We categorised 3995 patients into two groups: sBP $\geq 90 \mathrm{~mm} \mathrm{Hg}$ and warm profile group, and $\mathrm{sBP}<90 \mathrm{~mm} \mathrm{Hg}$ or cold profile group. In each group, patients were stratified across the use of inotropes within 24 hours of hospital presentation.

Primary and secondary outcomes The primary outcome was all-cause death throughout follow-up. Secondary outcomes included cardiovascular death throughout follow-up, all-cause death during index hospitalisation and after discharge, and HF hospitalisation.

Results A total of 793 patients (20\%) presented with $\mathrm{sBP}<90 \mathrm{~mm} \mathrm{Hg}$ or cold profile, whereas 3202 patients had $\mathrm{sBP} \geq 90 \mathrm{~mm} \mathrm{Hg}$ and warm profile; 276 patients (35\%) in the $\mathrm{sBP}<90 \mathrm{~mm} \mathrm{Hg} /$ cold group and 312 patients (10\%) in the $\mathrm{sBP} \geq 90 \mathrm{~mm} \mathrm{Hg} /$ warm group received initial inotropic treatment. Adjusted excess risk of inotrope use relative to no inotrope for the primary outcome measure was significant in the $\mathrm{sBP} \geq 90 \mathrm{~mm} \mathrm{Hg} /$ warm group (adjusted HR), 1.36; $95 \% \mathrm{Cl} 1.09$ to $1.72, \mathrm{p}=0.006$ ) but not in the $\mathrm{SBP}<90 \mathrm{~mm}$ $\mathrm{Hg} /$ cold group (adjusted HR, $1.28,95 \% \mathrm{Cl} 0.96$ to 1.69 , $\mathrm{p}=0.09$ ). Risk for postdischarge all-cause death and $\mathrm{HF}$ hospitalisation was not significantly different between the patients with inotropes and no inotropes in both groups.

\section{Strengths and limitations of this study}

- This study offers the current real-world patterns and outcomes of initial inotropic treatments in congestive heart failure.

- Data are derived from a large contemporary cohort in Japan.

- The observational nature of this study precludes the demonstration of a causal relationship between initial inotrope use and outcomes.

- Despite a careful and comprehensive attempt to correct the heterogeneity of the patient characteristics, unmeasured covariates may account for outcomes.

- The exact dose of inotropic agents and duration of their use were not available.

Conclusion Inotrope use in the absence of hypotension and hypoperfusion is still common, but associated with a worse long-term prognosis.

Trial registration number UMIN000015238.

\section{INTRODUCTION}

Congestive heart failure (HF) is a lifethreatening medical condition that often requires in-hospital management. Most hospitalised patients with HF are treated with diuretics to attenuate fluid overload, while others may receive intravenous vasodilators, which decrease ventricular filling pressure and relieve symptoms. ${ }^{1-5}$ On the other hand, inotropes can be used for patients with a severe reduction in cardiac output to maintain vital 
organ perfusion and blood pressure. However, previous studies have raised concerns regarding safety of inotrope use due to increased morbidity and mortality ${ }^{6-8}$ Inotropes, especially those with adrenergic mechanisms such as dobutamine and dopamine, can increase left ventricular afterload and cause tachycardia, myocardial ischaemia and arrhythmias. Non-catecholamine inotropes, such as milrinone, may increase the risk of hypotension. ${ }^{9}$ Thus, current American College of Cardiology (ACC) / American Heart Association (AHA) guideline states that the use of intravenous inotropic agents in hospitalised patients without documented low blood pressure or impaired perfusion is potentially harmful (Recommendation: class III, level of evidence: B). ${ }^{2}$ Current European Society of Cardiology (ESC) guideline also limits the use of inotropes to the patients with hypotension or to those with signs of impaired peripheral perfusion such as 'cold' HF profile (Recommendation: class III, level of evidence: A) ${ }^{1}$ However, decisions regarding the initial use of vasoactive agents still remains largely subject to clinician discretion. ${ }^{124}$ Despite an important decision making basis for the initiation of inotropes, few studies have considered the clinical signs of perfusion status such as the 'cold' or 'warm' profile for the evaluation of outcomes. ${ }^{10}$ In addition, most previous studies have evaluated the short-term morbidity and mortality of inotrope use, but data on longterm outcomes associating inotrope use are scarce. ${ }^{10}$

The aim of this study is to assess the current initial use of inotropic therapy and associated outcomes according to initial blood pressure and perfusion status in contemporary real-world practice in Japan.

\section{METHODS}

\section{Patient population}

The Kyoto Congestive Heart Failure (KCHF) registry is a physician-initiated, prospective, observational, multicentre cohort study that enrolled, without exclusion, all consecutive patients who were hospitalised for congestive HF between October 2014 and March 2016 in 19 participating hospitals in Japan. All patients enrolled in the KCHF registry had signs and symptoms of HF defined by modified Framingham criteria ${ }^{11}$ and required intravenous drugs within 24 hours of hospital presentation. For those patients with repeated HF hospitalisation during the study period, the first HF hospitalisation was selected as an index hospitalisation. The overall design of the KCHF study has been described in detail. ${ }^{13-15}$

All study procedures were conducted in compliance with the ethical principles of the Declaration of Helsinki. Clinical follow-up data were collected from hospital medical records or by contact with patients, their relatives or their referring physicians in October $2017 .^{16}$

\section{Definitions}

Inotrope use was defined as treatment with dopamine, dobutamine or phosphodiesterase inhibitors within
24 hours of hospital presentation. Norepinephrine was categorised as a vasopressor. Levosimendan was not available in Japan and was not included in this study. According to the systolic blood pressure (sBP) and data on the HF profile including 'warm' (ie, adequately perfused) and 'cold' (hypoperfused) on presentation, we stratified all patients into two groups: $\mathrm{sBP} \geq 90 \mathrm{~mm} \mathrm{Hg}$ /warm group, and $\mathrm{sBP}<90 \mathrm{~mm} \mathrm{Hg}$ / cold group.

\section{Outcomes}

The primary outcome was all-cause death throughout a 1-year follow-up. Secondary outcomes included all-cause death during index hospitalisation and all-cause death after discharge, cardiovascular death throughout the 1-year follow-up, and HF hospitalisation. The causes of death were classified according to the Valve Academic Research Consortium definitions and adjudicated by a clinical event committee. ${ }^{17}$

\section{Statistical analysis}

Categorical variables are expressed as numbers and percentages and are compared using the $\chi^{2}$ test. Continuous variables are expressed as means with SD or medians with IQRs and are compared using an analysis of variance or the Kruska-Wallis test depending on their distributions. The cumulative incidences of the primary and secondary outcome measures, except for all-cause death during hospitalisation, were estimated by the Kaplan-Meier method and compared using the log-rank test. We estimated HRs and $95 \%$ CIs with Cox's proportional hazards regression models. Proportional hazard assumptions for risk-adjusting variables were assessed on the plots of log (time) vs log [$\log$ (survival)] stratified by the variable, and verified to be acceptable. In the Cox's proportional hazards model, we simultaneously included the clinically relevant factors listed in table 1 as risk-adjusting variables and incorporated the centres as stratification variables. The risk for all-cause death during hospitalisation was assessed by a logistic regression model in which we constructed parsimonious models with the 11 clinically most relevant risk-adjusting variables listed in table 1 because of a small number of patients with outcome. Analyses of Cox's proportional hazards model with stratification were performed with SPSS V.19 (IBM). All other analyses were performed with JMP V.12.01 software (SAS Institute) and GraphPad Prism V.6.05 (GraphPad Software, La Jolla, California, USA). In this study, all reported $p$ values were two tailed, and $\mathrm{p}<0.05$ was considered to be significant.

\section{Patient and public involvement}

Patients or the public were not involved in the design, conduction, or reporting, or in the dissemination plans of our research.

\section{RESULTS}

Clinical characteristics

Among 3995 patients in this study (median age, 80 years; $1786(45 \%)$ women), 121 (3\%) had a sBP <90 mm Hg, 


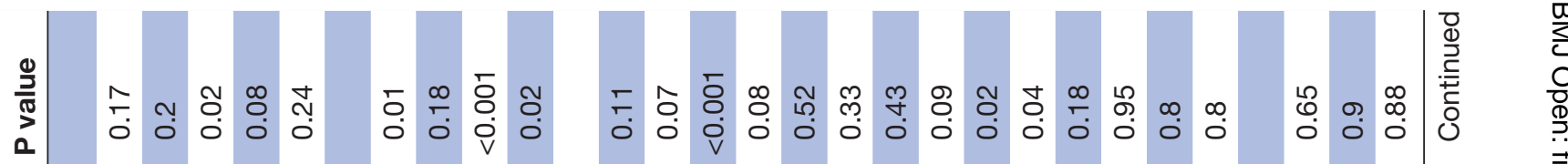

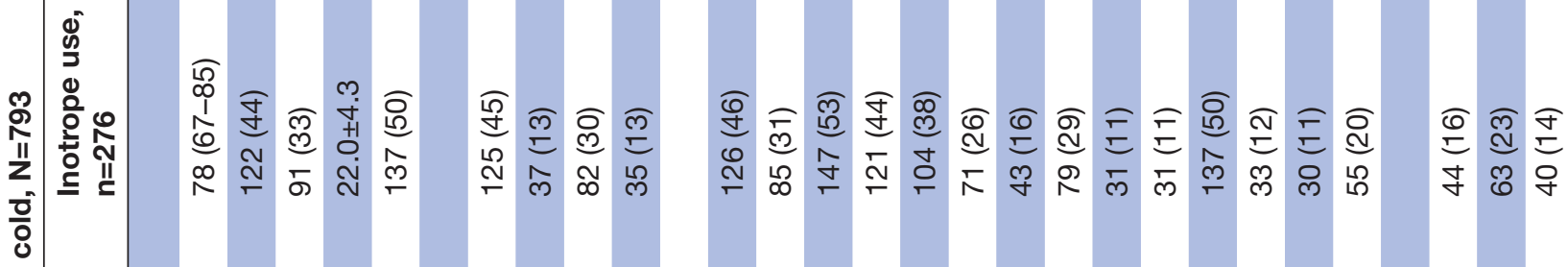

ᄒ

옫

है

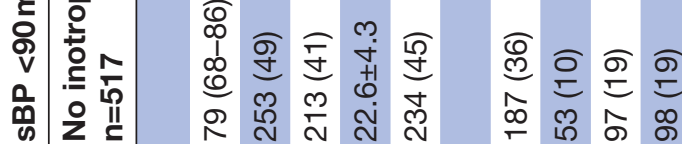

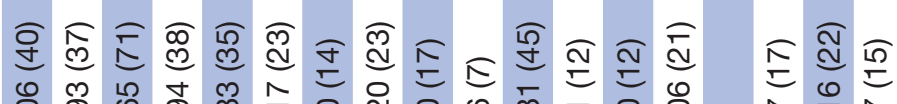

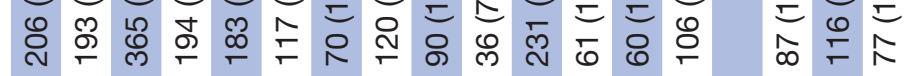

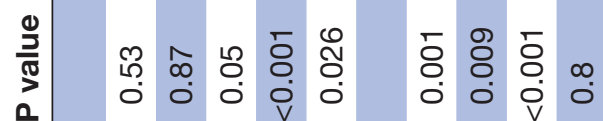

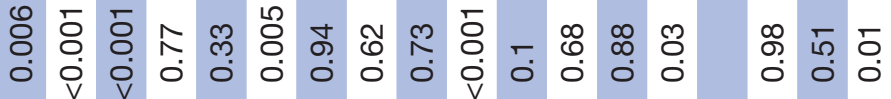

จิ 


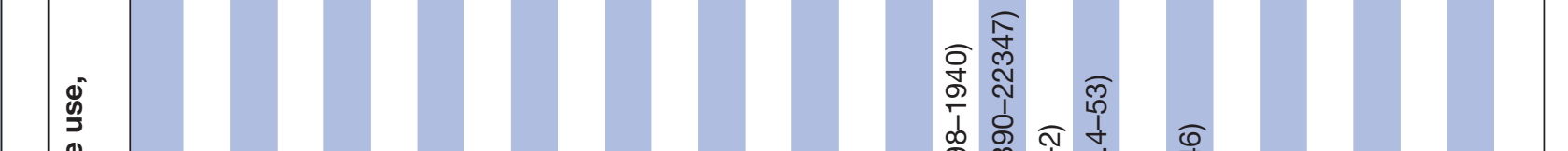

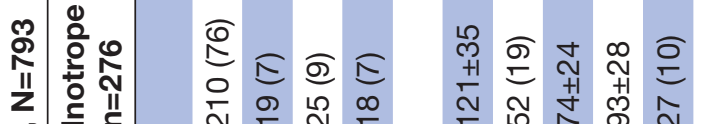

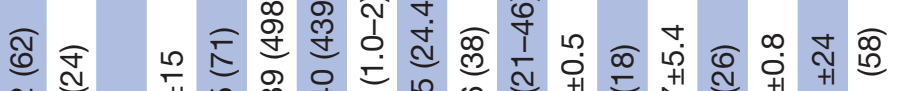

흥

ก ก

$\stackrel{1}{N} \underset{0}{N}$

岑

ᄒ

원

है

จิ

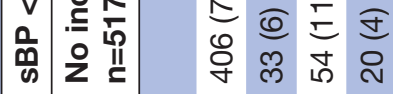

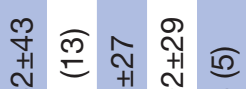

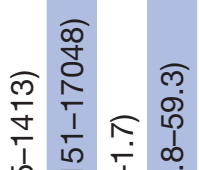

$\begin{array}{lll}\frac{0}{\pi} & & 0 \\ 0 & 0 \\ 0 & 0\end{array}$

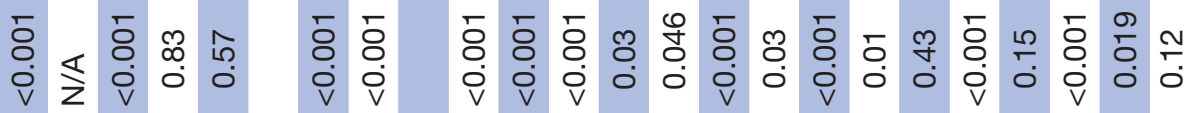

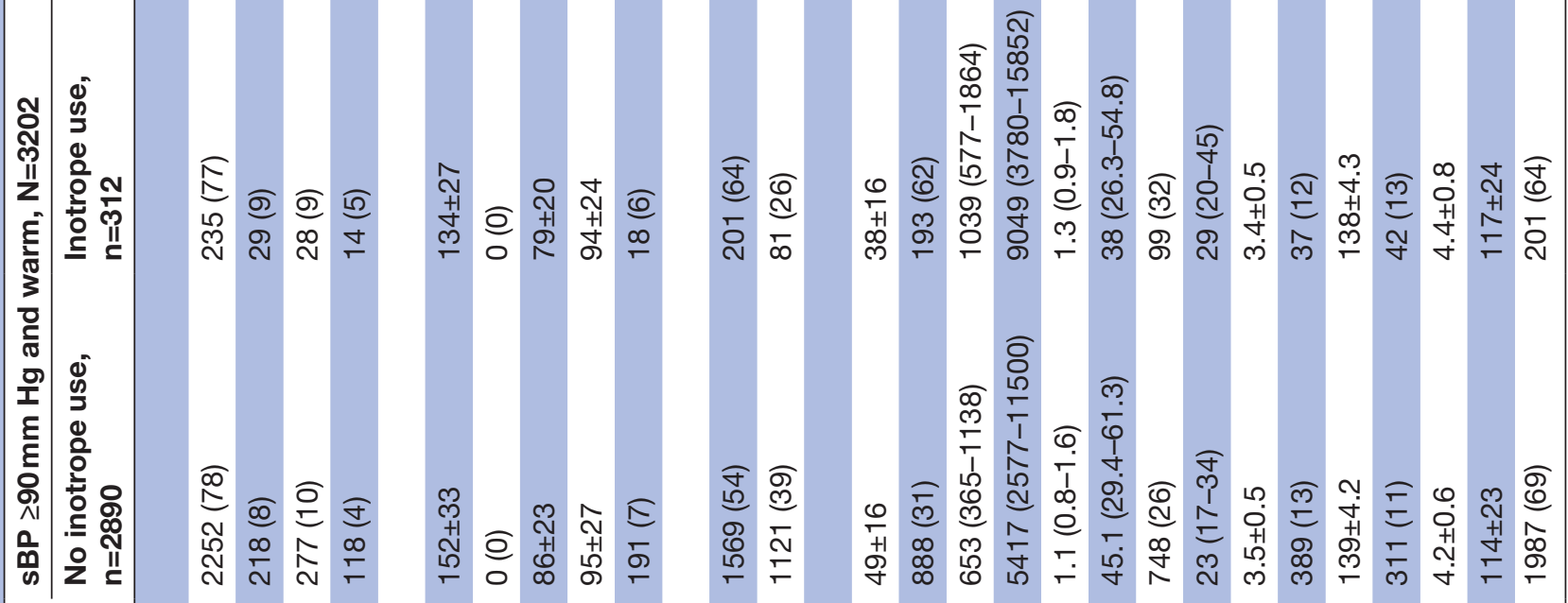

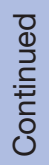

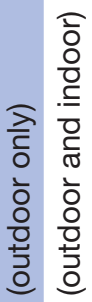

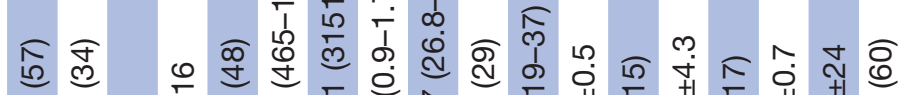

员

$\frac{\bar{c}}{\frac{9}{5}}$

$\stackrel{\mathbb{2}}{\circ}$ 
$\frac{2}{\frac{10}{3}}$

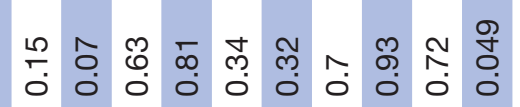

г.

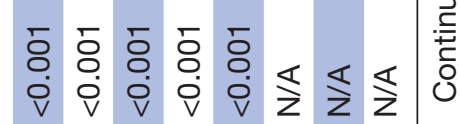

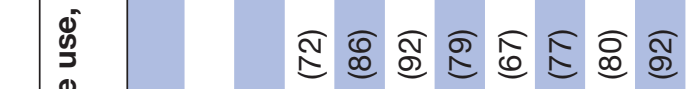

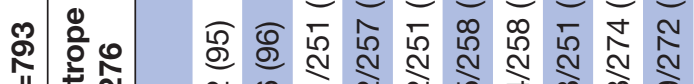

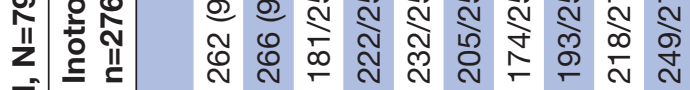

흥

$=$

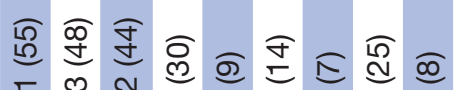

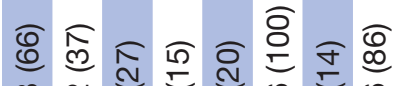

โ

品

ᄒ

올

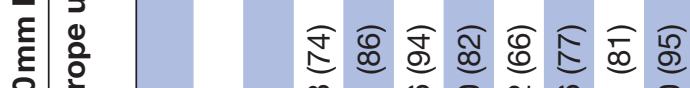

ชั่ำ สิ

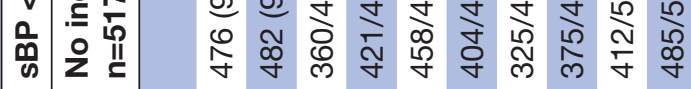

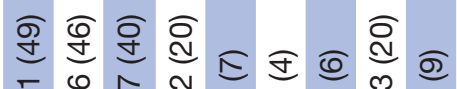

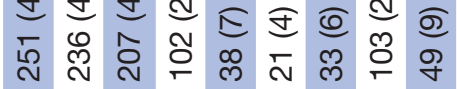

व ส্ర

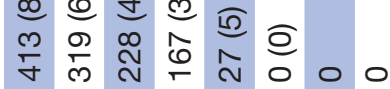

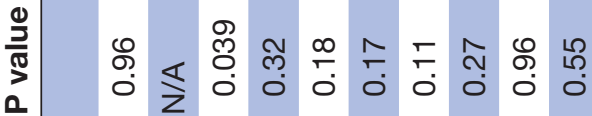

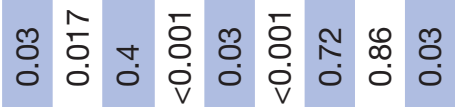

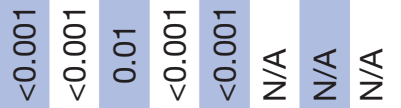

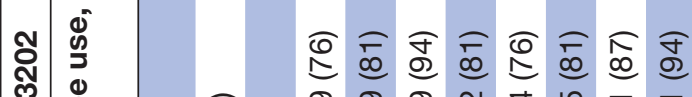

II

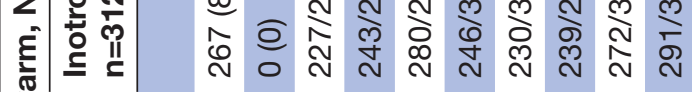

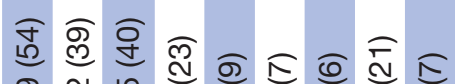

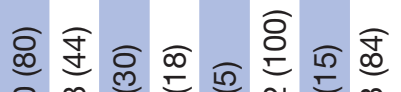

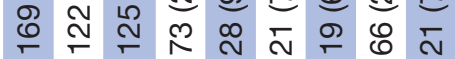

岕

3

든

ำ

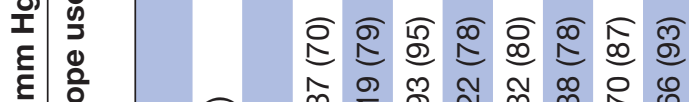

है।

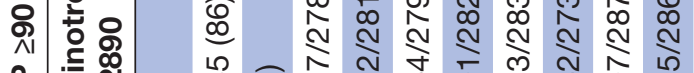

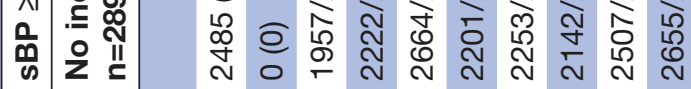

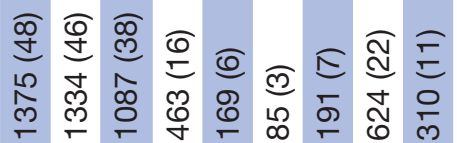

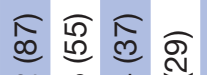

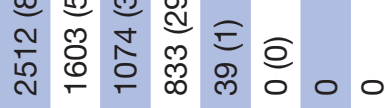

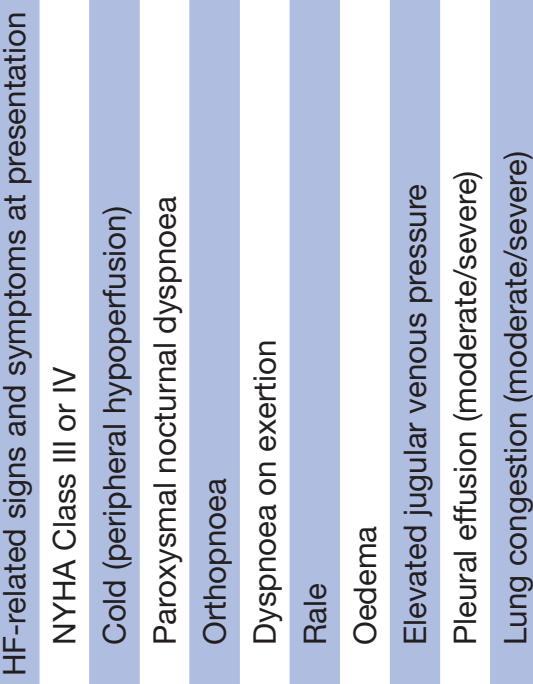

음

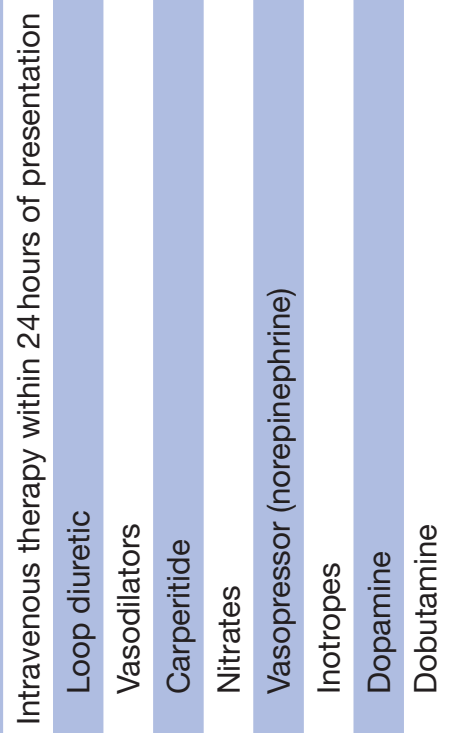




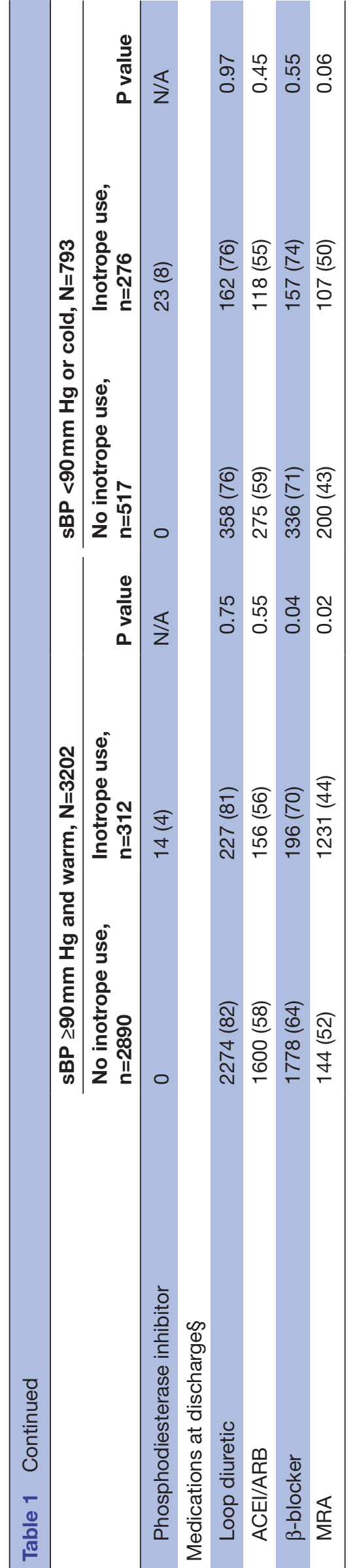

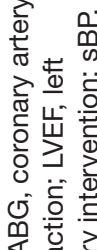
过苋 준
$748(19 \%)$ had a cold profile and $76(2 \%)$ had both a sBP $<90 \mathrm{~mm} \mathrm{Hg}$ and a cold profile on presentation. Accordingly, 793 patients (20\%) were categorised into sBP $<90 \mathrm{~mm} \mathrm{Hg}$ or cold group, whereas the remaining 3202 patients $(80 \%)$ were categorised into sBP $\geq 90 \mathrm{~mm}$ $\mathrm{Hg}$ and warm group. Overall, inotropes were used within 24 hours of hospital presentation in $588(15 \%)$ patients. Among those, 276 (47\%) were in the sBP $<90 \mathrm{~mm} \mathrm{Hg} /$ cold profile, whereas $312(53 \%)$ were in the sBP $\geq 90 \mathrm{~mm}$ $\mathrm{Hg}$ /warm group (figure 1). In both groups, the patients with inotrope use were more likely to have ischaemic heart disease and cardiomyopathy as a HF aetiology, have a history of ventricular tachycardia/fibrillation, a lower left ventricular ejection fraction, a higher brain natriuretic peptide level, and a lower estimated glomerular filtration rate compared with the patients without inotrope use (table 1). Regarding the vital signs at presentation, the patients with inotrope use had lower sBP and were less likely to have atrial fibrillation/flutter as an initial rhythm at hospital presentation in both the $\mathrm{sBP}<90 \mathrm{~mm}$ $\mathrm{Hg} /$ cold group and sBP $\geq 90 \mathrm{~mm} \mathrm{Hg} /$ warm group. Most of the study patients presented congestion-related signs and symptoms such as orthopnoea, dyspnoea on exertion, rales and elevated jugular venous pressure. The proportion of patients with congestion-related signs and symptoms was almost similar to the patients with inotrope use and no inotrope use in both groups (table 1). With respect to the medications at presentation, the proportion of patients receiving-blockers was not significantly different between the patients with and without inotrope use, whereas the proportion of patients receiving mineralocorticoid receptor antagonists were significantly higher in the patients with inotrope use compared with no inotrope use in both the sBP $<90 \mathrm{~mm} \mathrm{Hg}$ /hypoperfusion group and the $\mathrm{sBP} \geq 90 \mathrm{~mm} \mathrm{Hg}$ /warm group (table 1). Regarding the intravenous vasoactive agents used within 24 hours of presentation, more than half of the patients with no inotrope use and nearly $40 \%$ of the patients with inotrope use received intravenous vasodilators within 24 hours of presentation in both groups. Vasopressor (ie, norepinephrine) was more frequently used in the $\mathrm{sBP}<90 \mathrm{~mm} \mathrm{Hg} /$ hypoperfusion group than in the $\mathrm{sBP} \geq 90 \mathrm{~mm} \mathrm{Hg}$ /warm group. In both groups, it was more frequently used in the patients with inotrope use compared with no inotrope use. Overall, dobutamine was the most frequently used inotrope followed by dopamine and phosphodiesterase inhibitors (table 1). Patient characteristics across the three inotropic agents according to sBP and perfusion profile were shown in online supplemental table 1 .

\section{Long-term prognosis after admission}

The median follow-up was 463 days with a $94 \%$ follow-up rate at 1 year.

During the follow-up period, 823 patients $(26 \%)$ in the sBP $\geq 90 \mathrm{~mm} \mathrm{Hg} /$ warm group and 282 patients (36\%) in the $\mathrm{sBP}<90 \mathrm{~mm} \mathrm{Hg} /$ cold group died. In both groups, the cumulative 1-year incidence of all-cause death in the 


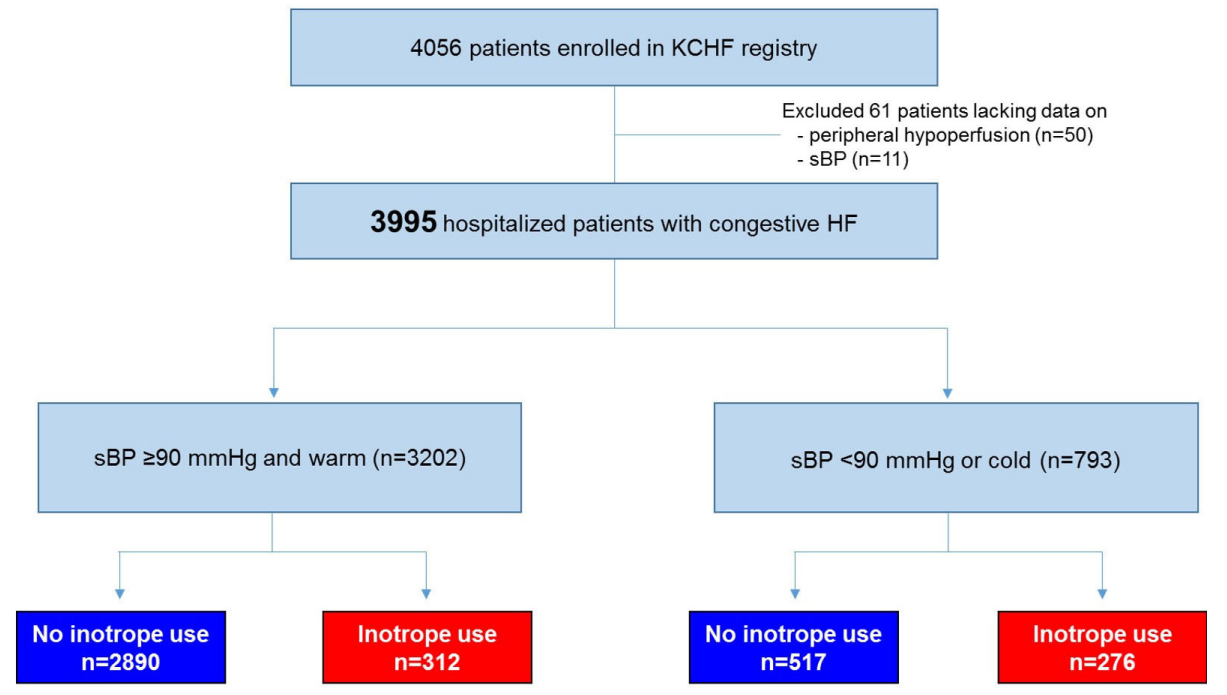

Figure 1 Study flow chart. HF, heart failure; KCHF, Kyoto Congestive Heart Failure; sBP, systolic blood pressure.

patients with inotrope use was significantly higher than in the patients without inotrope use (figure 2A,B). After adjustments for confounders, however, the risk of inotrope use relative to no inotrope use was significant only in the $\mathrm{sBP} \geq 90 \mathrm{~mm} \mathrm{Hg}$ /warm group, whereas adjusted excess risk of inotrope use relative to no inotrope use was not significant in the $\mathrm{sBP}<90 \mathrm{~mm} \mathrm{Hg}$ / cold group (table 2). Likewise, despite a significantly higher cumulative 1 year incidence of cardiovascular death in the patients with inotrope use relative to no inotrope use in both groups (online supplemental figure 1A,B), the adjusted excess risk of inotrope use relative to no inotrope use for cardiovascular death from admission was only significant in the $\mathrm{sBP} \geq 90 \mathrm{~mm}$ $\mathrm{Hg}$ /warm group but not in the $\mathrm{sBP}<90 \mathrm{~mm} \mathrm{Hg} /$ cold group (table 2). Even when use of norepinephrine was added to inotropes use as the inotrope/vasopressor use, adjusted excess risk of inotrope/vasopressor use relative to no inotrope/vasopressor use for the primary outcome measure was significant in the $\mathrm{sBP} \geq 90 \mathrm{~mm} \mathrm{Hg} /$ warm group (adjusted HR, 1.43, 95\% CI 1.16 to $1.77, \mathrm{p}<0.001$ ),

A

sBP $\geq 90 \mathrm{mmHg}$ and warm $(n=3202)$

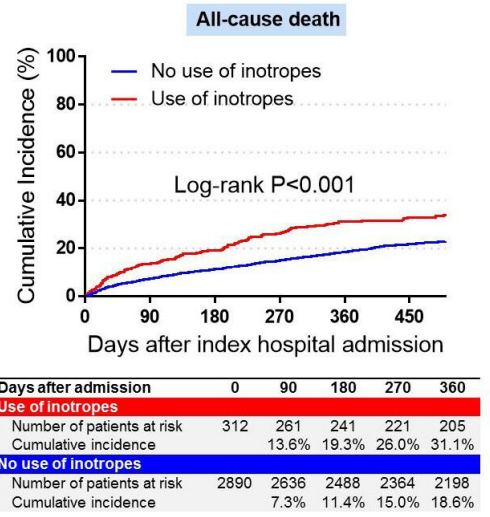

but not in the $\mathrm{sBP}<90 \mathrm{~mm} \mathrm{Hg}$ / cold group (adjusted HR, $1.30,95 \%$ CI 0.98 to $1.72, \mathrm{p}=0.07)$.

\section{In-hospital prognosis}

During index hospitalisation, 154 patients (5\%) in the $\mathrm{sBP} \geq 90 \mathrm{~mm} \mathrm{Hg}$ /warm group and 110 patients $(14 \%)$ in the $\mathrm{sBP}<90 \mathrm{~mm} \mathrm{Hg} /$ cold group died. In both groups, the incidence of all-cause death during index hospitalisation was significantly higher in the patients with inotrope use than that in those with no inotrope use (figure 3A,B). The adjusted excess risk of inotrope use relative to no inotrope use for all-cause death during index hospitalisation was significant in both groups (table 2).

\section{Postdischarge prognosis}

A total of 3048 patients in the $\mathrm{sBP} \geq 90 \mathrm{~mm} \mathrm{Hg}$ /warm group and 683 patients in the $\mathrm{sBP}<90 \mathrm{~mm} \mathrm{Hg} /$ cold group were discharged alive. In the $\mathrm{sBP} \geq 90 \mathrm{~mm} \mathrm{Hg} /$ warm group, 669 patients $(21 \%)$ died and 759 patients $(24 \%)$ were hospitalised for HF during the follow-up period, whereas

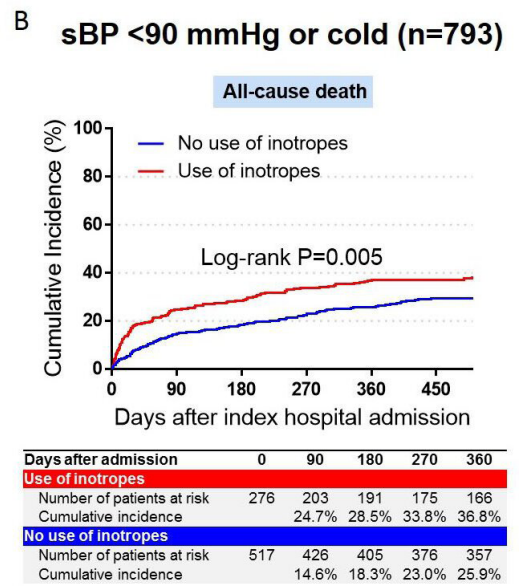

Figure 2 Kaplan-Meier curves for all-cause death across inotrope use according to sBP and perfusion profile. (A) patients with $\mathrm{sBP} \geq 90 \mathrm{~mm} \mathrm{Hg}$ and warm; (B) patients with $\mathrm{sBP}<90 \mathrm{~mm} \mathrm{Hg}$ or cold. sBP, systolic blood pressure. 
Table 2 Clinical outcomes across the patients with inotrope use and no inotrope use according to sBP and perfusion profile

\begin{tabular}{|c|c|c|c|c|c|c|}
\hline \multirow[b]{2}{*}{ Clinical outcomes } & \multicolumn{2}{|c|}{$\begin{array}{l}\text { In-hospital or cumulative } \\
1 \text {-year incidence (\%) }\end{array}$} & \multicolumn{4}{|c|}{ Inotrope use versus no inotrope use } \\
\hline & Inotrope use & $\begin{array}{l}\text { No inotrope } \\
\text { use }\end{array}$ & $\begin{array}{l}\text { Unadjusted HR } \\
(95 \% \mathrm{CI})\end{array}$ & $P$ value & $\begin{array}{l}\text { Adjusted HR* } \\
(95 \% \mathrm{Cl})\end{array}$ & $P$ value \\
\hline \multicolumn{7}{|l|}{$\mathrm{sBP} \geq 90 \mathrm{~mm} \mathrm{Hg}$ and warm, $\mathrm{n}=3202$} \\
\hline \multicolumn{7}{|l|}{ Primary outcome measure } \\
\hline All-cause death after admission & $94(31.1)$ & $524(18.6)$ & $1.54(1.25$ to 1.87$)$ & $<0.001$ & 1.36 (1.09 to 1.72$)$ & 0.006 \\
\hline \multicolumn{7}{|l|}{ Secondary outcome measures } \\
\hline $\begin{array}{l}\text { Cardiovascular death after } \\
\text { admission }\end{array}$ & $70(24.2)$ & $310(11.3)$ & 1.87 (1.46 to 2.37$)$ & $<0.001$ & 1.49 (1.13 to 1.94$)$ & 0.004 \\
\hline In-hospital all-cause death & $33(10.6)$ & $121(4.1)$ & 2.71 (1.78 to 4.01$)$ & $<0.001$ & 1.98 (1.28 to 3.07$)$ & 0.002 \\
\hline Post-discharge all-cause death & $61(22.9)$ & $421(15.7)$ & $1.32(1.03$ to 1.67$)$ & 0.03 & 1.25 (0.96 to 1.62$)$ & 0.10 \\
\hline HF hospitalisation & $76(30.3)$ & $577(22.7)$ & 1.35 (1.07 to 1.68$)$ & 0.01 & $1.26(0.99$ to 1.61$)$ & 0.07 \\
\hline \multicolumn{7}{|l|}{$\mathrm{sBP}<90 \mathrm{~mm} \mathrm{Hg}$ or cold, $\mathrm{n}=793$} \\
\hline \multicolumn{7}{|l|}{ Primary outcome measure } \\
\hline All-cause death after admission & $101(36.8)$ & $130(25.9)$ & $1.42(1.12$ to 1.80$)$ & 0.004 & 1.28 (0.96 to 1.69$)$ & 0.09 \\
\hline \multicolumn{7}{|l|}{ Secondary outcome measures } \\
\hline $\begin{array}{l}\text { Cardiovascular death after } \\
\text { admission }\end{array}$ & $75(28.9)$ & $91(18.7)$ & 1.49 (1.13 to 1.97$)$ & 0.006 & 1.35 (0.96 to 1.88$)$ & 0.08 \\
\hline In-hospital all-cause death & $63(22.8)$ & $47(9.1)$ & 2.96 (1.97 to 4.48$)$ & $<0.001$ & 2.27 (1.45 to 3.57$)$ & $<0.001$ \\
\hline Postdischarge all-cause death & 39 (19.7) & $88(19.0)$ & 0.93 (0.67 to 1.28$)$ & 0.68 & 0.88 (0.60 to 1.30$)$ & 0.52 \\
\hline HF hospitalisation & $54(27.7)$ & $108(25.5)$ & 1.15 (0.84 to 1.54$)$ & 0.38 & 1.10 (0.76 to 1.58$)$ & 0.62 \\
\hline
\end{tabular}

*Adjusted for the clinically relevant variables listed in table 1.

$\mathrm{HF}$, heart failure; sBP, systolic blood pressure.

172 patients (22\%) died and 186 patients $(24 \%)$ were hospitalised for $\mathrm{HF}$ in the $\mathrm{sBP}<90 \mathrm{~mm} \mathrm{Hg}$ /cold group. In the $\mathrm{sBP} \geq 90 \mathrm{~mm} \mathrm{Hg} /$ warm group, cumulative 1-year incidences of all-cause death after discharge and $\mathrm{HF}$ hospitalisation were significantly higher in the patients with inotrope use (figure 3C,E), whereas there was negligible difference between the patients with inotrope use and no inotrope use in the $\mathrm{sBP}<90 \mathrm{~mm} \mathrm{Hg} /$ cold group (figure 3D,F). After adjustment by confounders, excess risks of inotrope use relative to no inotrope use for allcause death after discharge and HF hospitalisation were not significant in both groups (table 2).

\section{DISCUSSION}

This study from a large contemporary Japanese cohort demonstrated that $15 \%$ of the patients hospitalised with congestive HF received inotropes within 24 hours of hospital presentation. Fifty-three per cent of those patients did not have the guideline-recommended clinical indications of inotrope use such as $\mathrm{sBP}<90 \mathrm{~mm} \mathrm{Hg}$ and a cold profile. Long-term mortality risk of initial inotrope use relative to no inotrope use was significant in the $\mathrm{sBP} \geq 90 \mathrm{~mm} \mathrm{Hg} /$ warm group, but not in the $\mathrm{sBP}<90 \mathrm{~mm}$ $\mathrm{Hg} /$ cold group. There were no differences in the risk for post-discharge all-cause death and HF hospitalisation between the inotrope use and no inotrope use in both
$\mathrm{sBP} \geq 90 \mathrm{~mm} \mathrm{Hg}$ /warm group and $\mathrm{sBP}<90 \mathrm{~mm} \mathrm{Hg} /$ cold group.

In addition to diuretics and vasodilators, inotropes have long been used for the treatment of decompensated HF, particularly for patients with cardiogenic shock or signs of impaired perfusion. However, previous studies demonstrated that the use of inotropes in the treatment of acute decompensated HF was associated with short-term adverse outcomes. ${ }^{6-8} 18$ Thus, US and European guidelines restrict the use of inotropic agents unless the patients have symptomatic hypotension or signs of peripheral hypoperfusion. ${ }^{12}$ Despite negative implications from the previous observational studies and guideline restrictions, our study demonstrated that inotrope use is still common in the current real world of hospitalised HF patients with $15 \%$ of the study participants receiving inotropes within 24 hours of hospital presentation. Notably, more than half of the patients initially treated with inotropes did not exhibit hypotension or signs of peripheral hypoperfusion on hospital presentation. The results were similar to those from the European Society of Cardiology Heart Failure Long-Term registry in which $12 \%$ of the study patients received inotropes and/or vasopressors during the first 24 hours after admission and $45.7 \%$ of these patients exhibited signs of peripheral hypoperfusion. ${ }^{10}$ In our study, crude 1-year mortality risk of the patients initially treated with inotropes was $31.1 \%$ in the $\mathrm{sBP} \geq 90 \mathrm{~mm} \mathrm{Hg}$ /warm group 
sBP $\geq 90 \mathrm{mmHg}$ and warm $(\mathrm{n}=3202)$

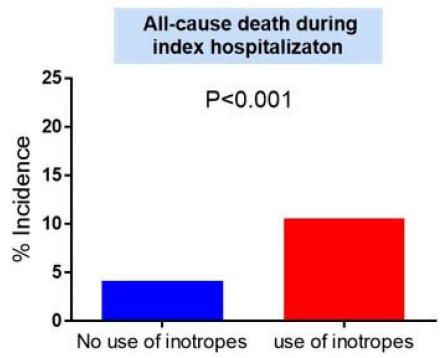

sBP $\geq 90 \mathrm{mmHg}$ and warm $(n=3048)$

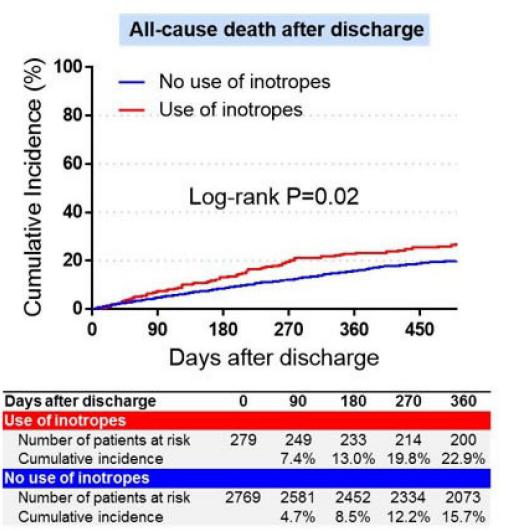

sBP $\geq 90 \mathrm{mmHg}$ and warm $(n=3048)$

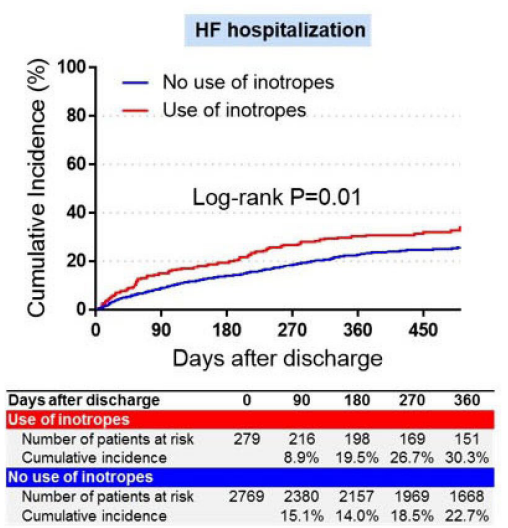

sBP $<90 \mathrm{mmHg}$ or cold $(\mathrm{n}=793)$

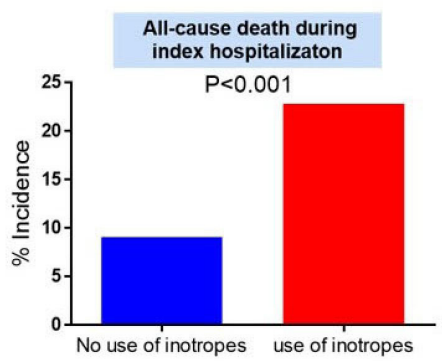

D

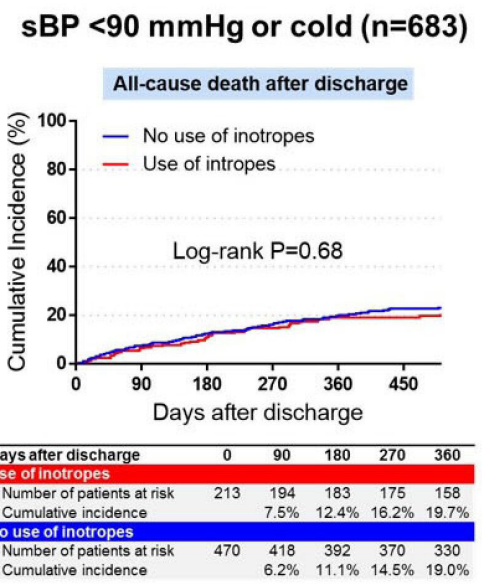

F

sBP $<90 \mathrm{mmHg}$ or cold $(n=683)$

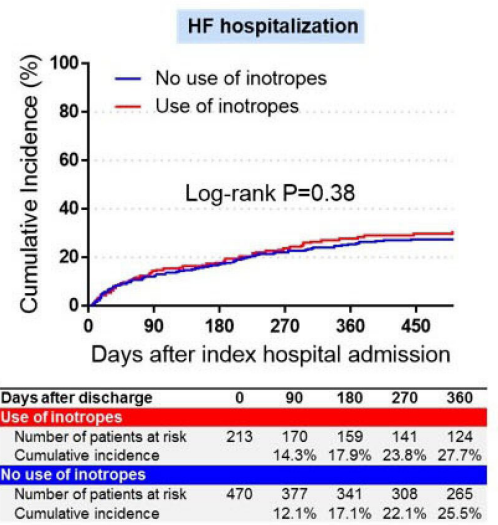

Figure 3 Incidence of all-cause death during index hospitalisation (A, B), all-cause death after discharge (C, D) and HF hospitalisation $(E, F)$. A, C and $E$, patients with $s B P \geq 90 \mathrm{~mm} \mathrm{Hg}$ and warm; B, D and F, patients with sBP $<90 \mathrm{~mm} \mathrm{Hg}$ or cold. $\mathrm{HF}$, heart failure; sBP, systolic blood pressure.

and $36.8 \%$ in the $\mathrm{sBP}<90 \mathrm{~mm} \mathrm{Hg} /$ cold group, both of which were much higher than that of the patients without inotrope use. However, inotrope use had a different effect size for longterm mortality across the two groups. Adjusted excess risk of inotrope use relative to no inotrope use for 1-year mortality was significant among the patients without hypotension or signs of impaired perfusion, whereas it was neutral among the patients presenting with hypotension or a cold profile. The results were similar with the previous reports by Kang et al showing that inotrope use was an independent predictor for in-hospital and 1-month postdischarge mortality in patients with initial $\mathrm{sBP} \geq 90 \mathrm{~mm} \mathrm{Hg}$, but not in patients with an initial 
$\mathrm{sBP}<90 \mathrm{~mm} \mathrm{Hg} .{ }^{19}$ However, the categorisation of our patients into the $\mathrm{sBP}<90 \mathrm{~mm} \mathrm{Hg}$ or cold group was mainly driven by 'cold' profile rather than sBP $<90 \mathrm{~mm} \mathrm{Hg}$ as more than $90 \%$ of this group had cold profile, whereas $85 \%$ had $\mathrm{sBP} \geq 90 \mathrm{~mm}$ $\mathrm{Hg}$. Given the limited options for medical management of decreased cardiac output and impaired peripheral organ perfusion, inotrope use might still be an option for patients with a cold profile. On the other hand, our results might support inappropriateness of inotrope use for haemodynamically stable patients with adequate peripheral perfusion, enforcing the current guidelines that discourage the routine use of inotropes. However, it should be noted that the present results should be interpreted carefully. Although the present results come from a large contemporary HF cohort, they provide only a snapshot of current real-world clinical practice. Its observational design per se precludes the demonstration of a causal relationship between initial inotropic treatment and outcomes. We cannot deny the possibility that the increased mortality associated with the use of inotropes may be attributed to patient characteristics rather than drugspecific characteristics.

A previous trial comparing the outcomes of dobutamine with nesiritide among patients with acute decompensated HF showed that even short-term infusion of dobutamine within 3 days resulted in a significantly higher 6 month mortality compared with nesiritide. ${ }^{20}$ Similarly, a 24-hour infusion of dobutamine was associated with a significantly higher 6-month mortality compared with levosimendan. These results suggest that even short-term exposure to catecholamines could affect long-term prognosis. ${ }^{21}$ Irreversible catecholamine-induced damage to cardiac myocytes has been suggested as an underlying mechanism..$^{21}$ However, we found that a high 1-year mortality risk in the inotrope stratum was mainly driven by a higher in-hospital mortality, whereas the risks of postdischarge mortality and HF hospitalisation were comparable between inotrope stratum and no inotrope stratum in both the $\mathrm{sBP} \geq 90 \mathrm{~mm} \mathrm{Hg} /$ cold and $\mathrm{sBP}<90 \mathrm{~mm} \mathrm{Hg} /$ warm groups. These results were in line with a recent observational study reporting a lack of association between inotrope use and 6 month post-discharge mortality in patients with reduced $\mathrm{EF}^{22}$ The results may suggest that adverse effects potentially induced by inotropes such as direct myocardial toxicity, increased oxygen consumption and arrhythmogenesis are temporal and do not last a long time. The results highlight unmet needs for the development of a more effective and safe treatment strategy targeting the initial phase of HF to overcome the imminent critical condition seen in our patients in the inotrope stratum.

\section{Limitations}

This study had several limitations. First, because of its observational nature, the decision to use vasoactive agents was made by the attending physicians, which is inherently subject to selection bias and confounding. Second, although detailed data in our dataset enabled intensive adjustment with most conceivable and previously established confounders, we were unable to exclude the influence of other unmeasured confounding factors.
In addition, for the evaluation of all-cause death during index hospitalisation, we used parsimonious models because of a small number of patients with outcome. Third, the exact dose of agents and the duration of their use were not available in our dataset. Thus, we were unable to distinguish low- and high-dose infusions of inotropes. Finally, although this study included nearly 4000 patients, the sample size was still insufficient to separately analyse the impact of individual drugs categorised as inotropes.

In conclusion, this study demonstrated the current use of inotropes in initial in-hospital treatment of HF in realworld practice. Inotropes are still commonly used against guideline recommendations. Inotrope treatment is associated with a high long-term mortality risk, particularly when used in the absence of shock or peripheral hypoperfusion. Development of standardised and evidence-based approaches to initial HF treatment might improve clinical outcomes.

\section{Author affiliations}

${ }^{1}$ Department of Cadiology, Osaka Red Cross Hospital, Osaka, Japan

${ }^{2}$ Department of Cardiovascular Medicine, Kyoto University Graduate School of Medicine, Kyoto, Japan

${ }^{3}$ Department of Cardiology, Mitsubishi Kyoto Hospital, Kyoto, Japan

${ }^{4}$ Department of Clinical Epidemiology, Hyogo College of Medicine, Nishinomiya, Japan

${ }^{5}$ Department of Cardiovascular Medicine, Shiga General Hospital, Moriyama, Japan ${ }^{6}$ Department of Cardiology, Tenri Hospital, Tenri, Japan

${ }^{7}$ Division of Heart Failure, National Cerebral and Cardiovascular Center, Suita, Japan ${ }^{8}$ Department of Cardiology, Hyogo Prefectural Amagasaki General Medical Center, Amagasaki, Japan

${ }^{9}$ Department of Cardiology, National Hospital Organization Kyoto Medical Center, Kyoto, Japan

${ }^{10}$ Department of Cardiology, Shimabara Hospital, Kyoto, Japan

${ }^{11}$ Department of Cardiology, Japanese Red Cross Otsu Hospital, Otsu, Japan

${ }^{12}$ Department of Cardiology, Hikone Municipal Hospital, Hikone, Japan

${ }^{13}$ Department of Cardiology, Kishiwada City Hospital, Kishiwada, Japan

${ }^{14}$ Department of Cardiology, Kansai Electric Power Hospital, Osaka, Japan

${ }^{15}$ Department of Cardiology, Shizuoka General Hospital, Shizuoka, Japan

${ }^{16}$ Department of Cardiology, Kurashiki Central Hospital, Kurashiki, Japan

${ }^{17}$ Department of Cardiology, Kokura Memorial Hospital, Kitakyushu, Japan

${ }^{18}$ Department of Cardiology, Japanese Red Cross Wakayama Medical Center, Wakayama, Japan

${ }^{19}$ Department of Cardiology, Nishi Kobe Medical Center, Kobe, Japan

${ }^{20}$ Cardiovascular Center, Tazuke Kofukai Medical Research Institute, Kitano Hospital, Osaka, Japan

${ }^{21}$ Department of Cardiovascular Medicine, Kobe City Medical Center General Hospital, Kobe, Japan

${ }^{22}$ Department of Cardiovascular Medicine, Shiga University of Medical Science, Otsu, Japan

${ }^{23}$ Department of Cardiovascular Medicine, Shinshu University Graduate School of Medicine, Matsumoto, Japan

Acknowledgements The authors thank the staff of the KCHF study and the members of the participating centres.

Contributors $\mathrm{KN}$ : conceptualisation, methodology, formal analysis, writing-original draft; TKato: conceptualisation, methodology, data curation, funding acquisition, interpretation, review, writing-review and editing, final approval, agreement to be accountable; HY, EY: data curation, investigation, resources; TMorimoto: methodology, formal analysis, writing-review and editing, supervision; YI, YT, YY, TKit, RT, Mlg, MaK, MTa, TJ, TIk, TKaw, AK, RN, YK, TMorinaga, KS, MiK, YSe, TIn, MIn, MTo, YF, YN, KA, KKa, SS, KO, YSa: investigation, resources; KKu, NO: investigation, resources, funding acquisition; TKim: project administration, methodology, supervision, writing-review and editing. TKato is the guarantor. 
Funding This study is supported by a grant from the Japan Agency for Medical Research and Development (18059186) to TK, KKu and NO.

Disclaimer The funder had no role in the study design, collection, analysis, or interpretation of data, writing the manuscript, or the decision to submit the paper for publication.

Competing interests None declared.

Patient consent for publication Not applicable.

Ethics approval This study was approved by Ethics Committees and reference number: Kyoto University Graduate School of Medicine (approval number: E2311), Shiga General Hospital (approval number: 20141120-01), Tenri Hospital (approval number: 640), Kobe City Medical Center General Hospital (approval number: 14094), Hyogo Prefectural Amagasaki General Medical Center (approval number: Rinri 26-32), National Hospital Organisation Kyoto Medical Center (approval number: 14-080), Mitsubishi Kyoto Hospital (approved November 12, 2014), Okamoto Memorial Hospital (approval number: 201503), Japanese Red Cross Otsu Hospital (approval number: 318), Hikone Municipal Hospital (approval number: 26-17), Japanese Red Cross 0saka Hospital (approval number: 392), Shimabara Hospital (approval number: E2311), Kishiwada City Hospital (approval number: 12), Kansai Electric Power Hospital (approval number: 26-59), Shizuoka General Hospital (approval number: Rin14-11-47), Kurashiki Central Hospital (approval number: 1719), Kokura Memorial Hospital (approval number: 14111202), Kitano Hospital (approval number: P14-11-012), Japanese Red Cross Wakayama Medical Center (approval number: 328 ).

Provenance and peer review Not commissioned; externally peer reviewed.

Data availability statement All data relevant to the study are included in the article or uploaded as online supplemental information.

Supplemental material This content has been supplied by the author(s). It has not been vetted by BMJ Publishing Group Limited (BMJ) and may not have been peer-reviewed. Any opinions or recommendations discussed are solely those of the author(s) and are not endorsed by BMJ. BMJ disclaims all liability and responsibility arising from any reliance placed on the content. Where the content includes any translated material, BMJ does not warrant the accuracy and reliability of the translations (including but not limited to local regulations, clinical guidelines, terminology, drug names and drug dosages), and is not responsible for any error and/or omissions arising from translation and adaptation or otherwise.

Open access This is an open access article distributed in accordance with the Creative Commons Attribution Non Commercial (CC BY-NC 4.0) license, which permits others to distribute, remix, adapt, build upon this work non-commercially, and license their derivative works on different terms, provided the original work is properly cited, appropriate credit is given, any changes made indicated, and the use is non-commercial. See: http://creativecommons.org/licenses/by-nc/4.0/.

\section{ORCID iDs}

Kazuya Nagao http://orcid.org/0000-0002-5043-8778

Takao Kato http://orcid.org/0000-0001-8213-7999

Takeshi Morimoto http://orcid.org/0000-0002-6844-739X

Moritake Iguchi http://orcid.org/0000-0001-5029-1203

Satoshi Shizuta http://orcid.org/0000-0003-4587-9031

Takeshi Kimura http://orcid.org/0000-0002-5665-4076

\section{REFERENCES}

1 Ponikowski P, Voors AA, Anker SD, et al. 2016 ESC Guidelines for the diagnosis and treatment of acute and chronic heart failure: The Task Force for the diagnosis and treatment of acute and chronic heart failure of the European Society of Cardiology (ESC) developed with the special contribution of the Heart Failure Association (HFA) of the ESC. Eur Heart J 2016;37:2129-200.

2 Yancy CW, Jessup M, Bozkurt B, et al. 2013 ACCF/AHA guideline for the management of heart failure: executive summary: a report of the American College of Cardiology Foundation/American
Heart Association Task Force on practice guidelines. Circulation 2013;128:1810-52.

3 O'Connor CM, Starling RC, Hernandez AF, et al. Effect of nesiritide in patients with acute decompensated heart failure. $N$ Engl $J$ Med 2011;365:32-43.

4 Tsutsui $\mathrm{H}$, Isobe M, Ito H, et al. JCS 2017/JHFS 2017 Guideline on diagnosis and treatment of acute and chronic heart failure- Digest Version. Circ J 2019;83:2084-184.

5 Mullens W, Damman K, Harjola V-P, et al. The use of diuretics in heart failure with congestion - a position statement from the Heart Failure Association of the European Society of Cardiology. Eur J Heart Fail 2019;21:137-55.

6 O'Connor CM, Gattis WA, Uretsky BF, et al. Continuous intravenous dobutamine is associated with an increased risk of death in patients with advanced heart failure: insights from the Flolan international randomized survival trial (first). Am Heart $J$ 1999;138:78-86.

7 Mebazaa A, Parissis J, Porcher R, et al. Short-term survival by treatment among patients hospitalized with acute heart failure: the global ALARM-HF registry using propensity scoring methods. Intensive Care Med 2011;37:290-301.

8 Abraham WT, Adams KF, Fonarow GC, et al. In-hospital mortality in patients with acute decompensated heart failure requiring intravenous vasoactive medications: an analysis from the acute decompensated heart failure national registry (adhere). J Am Coll Cardiol 2005;46:57-64.

9 Francis GS, Bartos JA, Adatya S. Inotropes. J Am Coll Cardiol 2014;63:2069-78.

10 Mebazaa A, Motiejunaite J, Gayat E, et al. Long-term safety of intravenous cardiovascular agents in acute heart failure: results from the European Society of cardiology heart failure long-term registry. Eur J Heart Fail 2018;20:332-41.

11 McKee PA, Castelli WP, McNamara PM, et al. The natural history of congestive heart failure: the Framingham study. N Engl J Med 1971;285:1441-6.

12 Senni M, Tribouilloy CM, Rodeheffer RJ, et al. Congestive heart failure in the community: a study of all incident cases in Olmsted County, Minnesota, in 1991. Circulation 1998;98:2282-9.

13 Yamamoto E, Kato T, Ozasa N, et al. Kyoto congestive heart failure (KCHF) study: rationale and design. ESC Heart Fail 2017;4:216-23.

14 Yaku H, Ozasa N, Morimoto T, et al. Demographics, management, and in-hospital outcome of hospitalized acute heart failure syndrome patients in contemporary real clinical practice in Japan- Observations from the prospective, Multicenter Kyoto Congestive Heart Failure (KCHF) Registry. Circ J 2018;82:2811-9.

15 Yaku H, Kato T, Morimoto T, et al. Association of mineralocorticoid receptor antagonist use with all-cause mortality and hospital readmission in older adults with acute decompensated heart failure. JAMA Netw Open 2019;2:e195892.

16 Japan's ethical guidelines for epidemiologic research. 2008, 2002. Available: http://www.lifescience.mext.go.jp/files/pdf/n796_01.pdf

17 Kappetein AP, Head SJ, Généreux P, et al. Updated standardized endpoint definitions for transcatheter aortic valve implantation: the valve academic research Consortium-2 consensus document. J Am Coll Cardiol 2012;60:1438-54.

18 Elkayam U, Tasissa G, Binanay C, et al. Use and impact of inotropes and vasodilator therapy in hospitalized patients with severe heart failure. Am Heart J 2007;153:98-104.

19 Kang J, Cho H-J, Lee H-Y, et al. Effects of widespread inotrope use in acute heart failure patients. J Clin Med 2018;7:368. doi:10.3390/ jcm7100368

20 Silver MA, Horton DP, Ghali JK, et al. Effect of nesiritide versus dobutamine on short-term outcomes in the treatment of patients with acutely decompensated heart failure. J Am Coll Cardiol 2002;39:798-803.

21 Follath F, Cleland JGF, Just $\mathrm{H}$, et al. Efficacy and safety of intravenous levosimendan compared with dobutamine in severe lowoutput heart failure (the LIDO study): a randomised double-blind trial. Lancet 2002;360:196-202.

22 Gomes C, Terhoch CB, Ayub-Ferreira SM, et al. Prognosis and risk stratification in patients with decompensated heart failure receiving inotropic therapy. Open Heart 2018;5:e000923. 